\section{Sorafenib prolongs survival in patients with advanced RCC}

Systemic therapies for patients with renal cell carcinoma (RCC) include high-dose interleukin 2 and interferon $\alpha$; however, these cytokines rarely induce complete response or prolonged survival and are not always well tolerated. Sorafenib, a multiprotein kinase inhibitor, has been shown to have antitumor activity in renal adenocarcinoma animal models and to prolong survival in patients with metastatic RCC. In a phase III trial, Escudier et al. showed that sorafenib prolonged survival in patients with advanced clear-cell RCC in whom previous therapy had failed.

The study included 903 patients (aged $\geq 18$ years) with clear-cell RCC who were randomly assigned to receive either placebo (arm A) or $400 \mathrm{mg}$ oral sorafenib twice daily (arm B). Both groups had similar baseline characteristics and the majority of participants had previously received cytokine-based therapy and had undergone nephrectomy. The average overall survival rates for patients in arm $A$ and arm B were 15.9 and 19.3 months, respectively $(P=0.02)$. Progression-free survival was also significantly extended after sorafenib treatment (5.5 months versus 2.8 months; $P<0.001$ ). The percentage of patients with partial response or stable disease was higher in the sorafenibtreated group than in the placebo group (10\% versus $2 \%$ and $74 \%$ versus $53 \%$, respectively; $P<0.001)$. The most frequent adverse events in the sorafenib-treated group were diarrhea, rash, fatigue, hand-foot skin reactions, alopecia, and nausea; cardiac ischemia and hypertension were also observed.

The authors conclude that oral sorafenib therapy prolongs progression-free survival in the subset of patients with advanced clearcell RCC who do not respond to first-line therapy.

Original article Escudier B et al. (2007) Sorafenib in advanced clear-cell renal-cell carcinoma. N Engl J Med 356: 125-134

\section{Sunitinib improves response and survival rates in metastatic RCC}

Patients with metastatic renal cell carcinoma (RCC) have low rates of response (5-20\%) to first-line therapy with interleukin 2 or interferon $\alpha$; median overall survival is approximately 12 months. The multityrosine kinase inhibitor sunitinib malate targets the overexpression of vascular endothelial growth factor receptor (VEGFR) and platelet-derived growth factor receptor (PDGFR) that occurs in many cases of RCC through inactivation of the VHL gene, and has shown promise in cytokine-resistant patients. Data from a phase III trial provide further evidence that angiogenic inhibition is promising as a treatment strategy for clear-cell RCC.

Motzer et al. randomized 750 patients with metastatic clear-cell RCC to first-line treatment with sunitinib $(50 \mathrm{mg}$ orally once daily for 4 weeks, then 2 weeks without treatment, in 6-week cycles) or interferon $\alpha$ (titrated to $9 \mathrm{MU}$ subcutaneously 3 times a week). Patients in the sunitinib group had significantly longer median progression-free survival than those on interferon a ( 11 vs 5 months; $P<0.001)$. Sunitinib also resulted in a significantly increased objective response rate $(31 \%$ vs $6 \% ; P<0.001)$. Symptoms including diarrhea and grade 3 or 4 neutropenia were more frequently reported in the sunitinib group, but patients on this treatment reported a significantly better quality of life than did patients on interferon $\alpha(P<0.001)$, and most adverse events resolved upon dose interruption or modification. Long-term data are needed to clarify issues such as the role of angiogenic growth factors in metastatic RCC, and the relative survival benefits of sunitinib and interleukin 2.

Original article Motzer RJ et al. (2007) Sunitinib versus interferon alfa in metastatic renal-cell carcinoma. $N$ Engl J Med 356: 115-124

\section{Trastuzumab after adjuvant chemotherapy: survival benefit in HER2-positive breast cancer}

Administration of trastuzumab after chemotherapy has been shown to improve diseasefree survival in patients with HER2-positive early-stage breast cancer. The Herceptin Adjuvant (HERA) study investigators have explored whether this humanized monoclonal antibody, which targets HER, also improves overall survival. Results after 2 years of follow-up are positive, showing a considerable overall survival benefit for women receiving trastuzumab. 